\title{
Seizure, mental retardation and abnormal cranial CT in a child
}

\author{
KS Anand, A Biswas, S Singh, A Prasad
}

A five-year-old boy presented with generalised tonic-clonic seizures since the age of three months. He was the first of two siblings born of nonconsanguinous marriage. He was born at full term by normal vaginal route and at that time his mother was 32 and father 34 years of age. His motor and mental milestones were delayed. There was no history of meningo-encephalitis or head injury. There was no family history of seizure disorder.

The general physical examination revealed prominence of the medial epicanthal folds, hypertelorism, short stature (height $=85 \mathrm{~cm}$ ), stubby hands, simian crease, clinodactyly, low set ears, thick lips and macroglossia. Neurological and other systemic examinations were unremarkable except for severe mental retardation and generalised hypotonia. A basic work-up involving haemogram, urinalysis, liver function, serum creatinine, muscle enzymes and lipid profile was normal. Serum calcium, phosphorus and alkaline phosphatase were $2.25 \mathrm{mmol} / 1,1.1 \mathrm{mmol} / 1$ and $78 \mathrm{IU} / \mathrm{l}$, respectively; $24-\mathrm{h}$ urinary calcium was $1.5 \mathrm{mmol} / 24 \mathrm{~h}$. The electrocardiogram and a $2-\mathrm{D}$ echocardiogram were normal. A nonenhanced cranial computed tomography (CT) scan is shown in the figure.

Department of

Neurology,

Institute of Human

Behaviour and Allied

Sciences,

Jhilmil, GT Road,

Shahdara,

Delhi-110095, India

K S Anand

A Biswas

S Singh

A Prasad

Accepted 9 July 1997

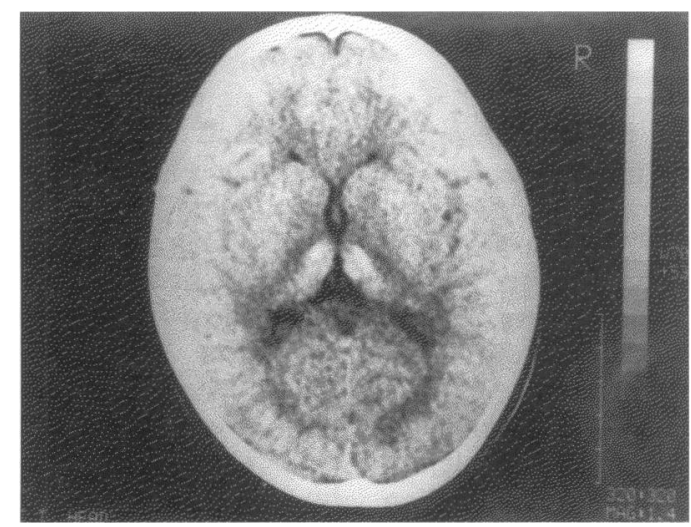

\section{Questions}

1 What does the CT scan show?

2 What is the probable aetiology in this case? 3 What are the differential diagnoses?

Figure Nonenhanced cranial CT scan 
Answers

QUESTION

Cranial CT shows bilateral symmetrical hyperdense areas over the thalamus. This finding is consistent with bilateral thalamic calcification

QUESTION 2

The child had multiple stigmata of Down's syndrome. In fact, his karyotyping revealed 47 chromosomes, chromosome 21 being repeated three times (trisomy) rather than twice. Basal ganglia calcification is found with increasing frequency in Down's syndrome. ${ }^{1}$ This has been considered to be due to genetic or non-genetic causes, such as anoxia and metabolic insults to the brain. ${ }^{2}$

QUESTION 3

Basal ganglia calcification is associated with a broad spectrum of clinical states. A disorder of the parathyroid gland with altered calcium and phosphorus metabolism accounts for $40-80 \%$ of cases. ${ }^{3}$ Decreased secretion of biologically active hormone or defective organ sensitivity to parathyroid hormone are often associated with basal ganglia calcification. It is also associated with various infections, enzymatic and biochemical defects and neurodegenerative conditions.

\section{Discussion}

Basal ganglia calcification is uncommon in children in whom it mostly follows necrosis of neural tissues by toxins, metabolic defects, infections or physical agents. Wisniewski et al first provided a comprehensive analysis of basal ganglia calcification in Down's syndrome. Although the random population frequency of basal ganglia calcification detection by CT scan is $0.3-0.6 \%$, it is detected in as many as $26.6 \%$ of Down's syndrome cases. ${ }^{1}$ Calcium deposits in basal ganglia have been reported on histopathological examination in $100 \%$ of postmortem specimens of Down's syndrome patients, which suggests that the condition is characterised by an unusual propensity for developing this pathologic manifestation. The

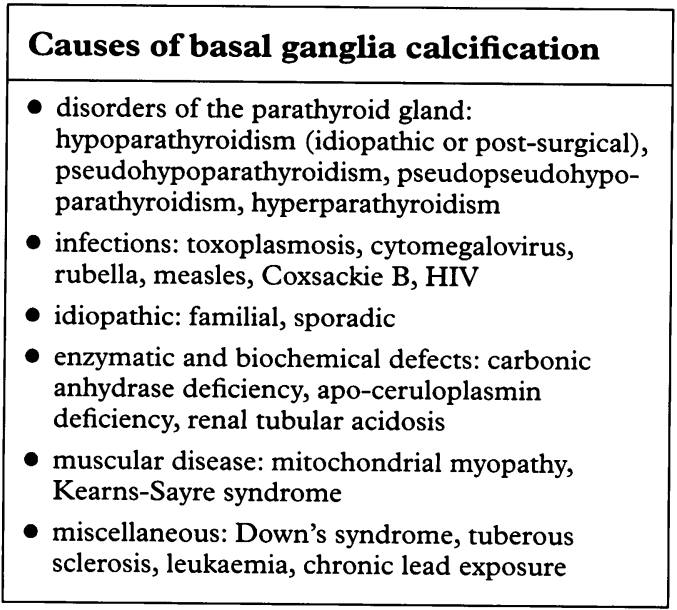

\section{Learning points}

- patients with Down's syndrome are more prone to develop basal ganglia calcification

- symptomatology of basal ganglia calcification presents only in a minority of cases with epilepsy, mental retardation, psychiatric abnormalities, EEG abnormalities, extrapyramidal syndromes, neuro-endocrinologic dysfunction, etc

- the diagnosis of basal ganglia calcification is a neuroradiologic rather than a clinical one

- the pathogenesis of basal ganglia calcification in Down's syndrome is uncertain, but premature aging, various cardiovascular and biochemical abnormalities may be responsible for cerebral calcification

Box 2

high frequency of basal ganglia calcification in Down's syndrome has been considered to be due to causes such as anoxia and metabolic insults to the brain. ${ }^{2}$

Vascular and circulatory anomalies are known to have an essential role in cerebral calcification and also occur with great frequency in Down's syndrome. ${ }^{2}$ Cardiac malformations were described in $75 \%$ of Down's syndrome patients who died before one year of age and in $35 \%$ of those who lived beyond one year of age. ${ }^{4}$ Various circulatory anomalies include hypoplastic cerebral vascular system, atherosclerosis and moya-moya disease. ${ }^{2}$ These vascular and circulatory anomalies produce reduced cerebral oxygenation and could explain the vulnerability of basal ganglia for calcification, as they are located within the border zones or 'watershed areas' and these bear the brunt of any hypoxic insult. ${ }^{3}$ In autopsy specimens of brainstem of Down's syndrome patients, Murofushi defined the deposited material as pseudo-calcium, based on its histologic properties under specific staining procedures and he related its occurrence to chronic anoxia, probably secondary to circulatory insufficiency. ${ }^{5}$

Although there are various biochemical abnormalities found in Down's syndrome, it is still uncertain whether they play any role in basal ganglia calcification. Impaired mucopolysaccharide metabolism has been described in cases of Down's syndrome as well as in cerebral calcification $^{2}$ and this could be an important link between the two.

For unknown reasons, basal ganglia calcification is a frequent finding in many disorders associated with hypocalcaemia. A decreased serum total calcium has been stated to be present in Down's syndrome subjects, in studies performed prior to currently available measures of serum ionised calcium. ${ }^{1}$

Due to the lack of evidence of disturbance of calcium metabolism and high frequency of hydrocephalus ex vacuo secondary to brain atrophy in their patients, Wisniewski et al concluded that basal ganglia calcification was the result of accelerated aging of the brain in Down's syndrome.

In our case there were no cardiac or gross vascular anomalies. There were no 
disturbances in calcium metabolism nor do we have proof of impaired mucopolysaccharide metabolism to provide some aetiological clues for the basal ganglia calcification. Thus, we must await a more definitive explanation before drawing any conclusions regarding the pathogenesis of basal ganglia calcification in Down's syndrome.

1 Wisniewski KE, French JH, Rosen JF, Koslowski PB, Tenner M, Wisniewski HM. Basal ganglia calcification (BGC) in Down's syndrome. Another manifestation of premature aging. Ann NY Acad Sci 1982;396:179-89.

2 Jakab I. Basal ganglia calcification and psychosis in mongolism. Eur Neurol 1978;17:300-14.

3 Lowenthal A, De Deyn P. Striopallidodentate calcifications. In: Joseph AB, Young RR, eds. Movement disorders in neurolIn: Joseph AB, Young RR, eds. Movement disorders in neurol-
ogy and neuropsychiatry. Oxford: Blackwell Scientific Publiogy and neuropsychiatry.

\section{Final diagnosis}

Bilateral basal ganglia calcification in Down's syndrome.

Keywords: Down's syndrome; calcification; basa ganglia

\footnotetext{
4 Benda CE. The child with mongolism (congenital acromicria). New York: Grune \& Stratton, 1960.

5 Murofushi K. Symmetrischer Pseudokalk in stammganglien and Grosshirnmark mit diskreter Leukencephalopathie bei Downschem syndrom. Neuropediatrie 1974;5 103-8.
}

Poole Hospital NHS

Trust,

Longfleet Road,

Poole,

Dorset BH15 2JB, UK

S Zaman

M Thomas

The Royal

Bournemouth

Hospital,

Castle Lane East,

Bournemouth,

Dorset BH7 7DW, UK

J Mallya

Correspondence to

Dr M Thomas

Accepted 25 June 1997

\section{A man with purple toes}

\author{
Syed Zaman, Jagadish Mallya, Matthew Thomas
}

A 79-year-old man was admitted for urgent angiography because of pain and discolouration of his toes. Six months prior to admission he was commenced on amiodarone and warfarin for paroxysmal atrial fibrillation. He was known to have hypercholesterolaemia, ischaemic heart disease and congestive cardiac failure. He was an ex-smoker. His other medications were enalapril, frusemide and cholestyramine.

Examination revealed purple discolouration of his toes with evidence of digital infarction (figures 1 and 2), blood pressure was $140 / 80 \mathrm{mmHg}$ and no murmurs or bruits were audible. Lower limb pulses were all palpable and Doppler pressure studies suggested small vessel occlusion without significant large vessel disease.

Investigations revealed: haemoglobin $12.2 \mathrm{~g} / \mathrm{dl}$, platelets $337 \times 10^{9} / 1$, erythrocyte sedimentation rate (ESR) $36 \mathrm{~mm} / \mathrm{h}$, white blood cells $6.9 \times 10^{9} / 1$ (neutrophils 4.5 , lymphocytes 1.5 , monocytes 0.5 , eosinophils 0.1 , basophils 0.1 ). Na $141 \mathrm{mmol} / 1, \mathrm{~K} 4.7 \mathrm{mmol} / 1$, urea $12.1 \mathrm{mmol} / 1$ creatinine $169 \mathrm{mmol} / 1$, cholesterol $3.6 \mathrm{mmol} / 1$, auto-immune profile and ANCA negative. Electrocardiogram showed sinus rhythm, left ventricular hypertrophy, anteroseptal $Q$ waves. Echocardiography showed impaired left ventricular function. Abdominal ultrasound showed a 4-cm abdominal aortic aneurysm.

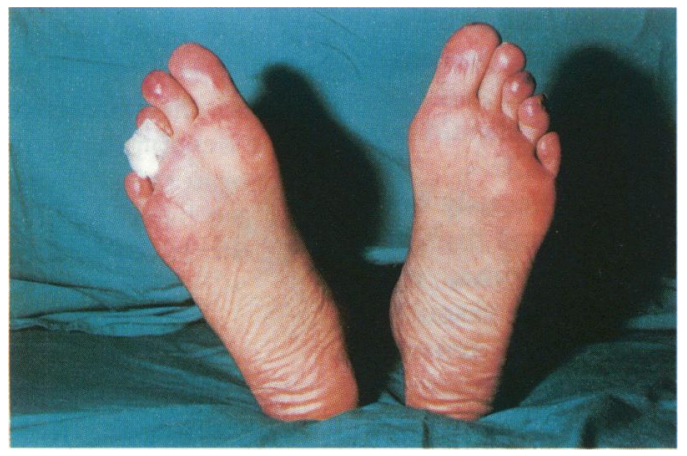

Figure 1

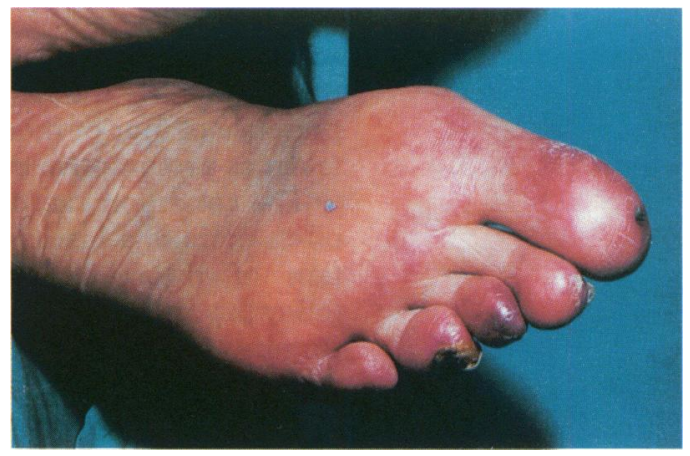

Figure 2

\section{Questions}

1 Give the most probable diagnosis and list the risk factors for the condition.

2 List five other clinical manifestations of this disorder.

3 Comment on management and prognosis. 